ISSN 0258-7122

Bangladesh J. Agril. Res. 34(3) : 465-472, September 2009

\title{
HETEROSIS IN BOTTLE GOURD
}

\author{
A.K.M. QUAMRUZZAMAN, M.A. RASHID \\ M.A.T. MASUD AND M. NAZIM UDDIN
}

\begin{abstract}
Heterosis in bottle gourd was studied in a set of $13 F_{1}$ with 26 parents. Results indicated highly significant differences for all the characters among the materials studied. Heterosis was higher for yield per plant, number of fruits per plant and individual fruit weight, medium in fruit length and fruit diameter, and lower in days to 1st harvest. Hybrids $\left(\mathrm{F}_{1}\right) 10 \times 17$ and $19 \times 26$ manifested highest heterosis over midparent (73.1\%) and better parent (61.8\%), respectively, for yield per plant.
\end{abstract}

Key Words : Heterosis, bottle gourd, hybrid.

\section{Introduction}

Bottle gourd [Lagenaria siceraria (Mol.) Standl.] is one of the most important and widely cultivated popular winter vegetable in Bangladesh. It occupies about 12,100 ha with a total production of 101,325 tons. The average yield is only 8.37 tons per hectare (Anon., 2005), which is very low as compared to that in other tropical countries. This low yield may be either due to lack of high yielding varieties or poor fertility management. High yielding variety is an important factor for maximizing the yield of bottle gourd. Though a large number of bottle gourd varietiesl genotypes are available in this country for cultivation, most of them lost their potentiality due to cross pollination occurs during the pollination. There is a great scope for the improvement of this crop through hybridization. Therefore, the present study was undertaken to develop high yielding $F_{1}$ varieties suitable for winter as well as summer rainy season cultivation and to study the heterosis in bottle gourd.

\section{Materials and Method}

The experiment was conducted at the farm of Olericulture Division, Horticulture Research Centre, Bangladesh Agricultural Research Institute, Gazipur, Bangladesh during the winter season of 2004-05. The seeds of $13 F_{1}$ with 26 parents of bottle gourd were sown on the polybag on 15 October 2004. The experiment was laid out in RCB design with three replications. The unit plot size was $8.0 \times 2.0 \mathrm{~m}$ maintaining $2.0 \times 2.5 \mathrm{~m}$ spacing. The land was fertilized with cowdung, urea, TSP, MP, gypsum, and zinc @ 15000, 175, 175, 150, 100, and 12 $\mathrm{kg} / \mathrm{ha}$, respectively. The total amount of cowdung, TSP, gypsum, and zinc and

Olericulture Division, Horticulture Research Centre (HRC), BARI, Gazipur-1701, Bangladesh. 
$1 / 3^{\text {rd }}$ of each of urea and MP were applied during fmal land preparation and in the pit. The rest of urea and MP were applied in four equal installments at 21, 35, 55, and 75 days after transplanting. The 25 days old seedlings were transplanted in the main field on 10 November 2004. The intercultural operations (weeding, irrigation, insecticide spray, etc.) were done as and when necessary. Data on days to first harvest, fruit length $(\mathrm{cm})$, fruit diameter $(\mathrm{cm})$, fruit number per plant, individual fruit wt $(\mathrm{kg})$, fruit yield (kg/plant) were recorded from three randomly selected plants per entry per replication. The data on different characters was statistically analyzed. The significance of increase or decrease in $F_{1}$ hybrid over their corresponding mid parent and better parent was tested by comparing their means with the help of appropriate standard error values in percentage (Table 1).

\section{Results and Discussion}

The mean performance for $13 \mathrm{~F}_{1}$ with 26 parents and per cent heterosis over mid parent and better parent for yield and yield contributing characters of heterotic crosses are presented in Table 1.

In case of days to first harvest six hybrids showed significant negative heterosis over mid parent indicating earliness. The estimates of mid parent heterosis ranged from -13.4 to $7.2 \%$. The highest significant negative heterotic response for earliness was observed in the hybrid $22 \times 29$ followed by $6 \times 13$ ($5.8 \%$ ). Nine hybrids performed significant negative better parent heterosis. The estimates of better parent heterosis ranged from -0.9 to -13.8 percent. The highest negative heterotic effect was also observed in the hybrid $22 \times 29$ followed by $6 \times$ 13 (-7.9\%). Singh et al. (1988) reported significant heterosis over the better parent was ARBGH-7 x LC2-1 for days to first female flowering and PSPL $\mathrm{x}$ ARBGH-7 for days to first fruit harvest. Sit and Sirohi (2002) also observed that the parental populations and hybrids were significantly different with regard to flowering in bottle gourd.

Among the 13 hybrids, seven hybrids showed significant positive heterotic response over mid parent for fruit length and range was 7.7-30.9\%. Only one hybrid showed more than $20 \%$ heterosis. Highest percent mid parent heterosis was observed from the hybrid $10 \times 17$ followed by 23 x 30 (18.8\%). For better parent, heterosis only four hybrids showed significant positive response in which three hybrids showed more than 20\% heterosis. Significant positive heterosis for fruit length in bottle gourd was found by Singh et al. (1998) and Sit and Sirohi (2002). In case of fruit diameter, only one cross (22x29) exhibited more than $20 \%$ mid parent and better parent heterosis. Singh et al. (1998) and Sit and Sirohi (2002) also observed significant positive heterosis in fruit diameter of bottle gourd. 
Table 1. Mean performance and percent heterosis over mid parent and better parent for yield and yield contributing characters in bottle gourd.

\begin{tabular}{|c|c|c|c|c|c|c|c|c|c|}
\hline \multirow{2}{*}{$\begin{array}{l}\text { Crosses/ } \\
\text { Parents }\end{array}$} & \multirow{2}{*}{$\begin{array}{c}\text { Mean } \\
\text { performancee } \\
\text { (days) }\end{array}$} & \multicolumn{2}{|c|}{ Days to first harvest } & \multirow{2}{*}{$\begin{array}{c}\text { Mean } \\
\text { performance } \\
(\mathrm{cm})\end{array}$} & \multicolumn{2}{|c|}{ Fruit length } & \multirow{2}{*}{$\begin{array}{c}\text { Mean } \\
\text { performance } \\
(\mathrm{cm})\end{array}$} & \multicolumn{2}{|c|}{ Fruit diameter } \\
\hline & & $\begin{array}{c}\text { Mid } \\
\text { parent } \\
\end{array}$ & $\begin{array}{l}\text { Better } \\
\text { parent }\end{array}$ & & $\begin{array}{c}\text { Mid } \\
\text { parent }\end{array}$ & $\begin{array}{l}\text { Better } \\
\text { parent }\end{array}$ & & $\begin{array}{c}\text { Mid } \\
\text { parent }\end{array}$ & $\begin{array}{l}\text { Better } \\
\text { parent }\end{array}$ \\
\hline $06 \times 13$ & 105 & $-5.8^{* *}$ & $-7.9 * *$ & 37.5 & -0.7 & -3.8 & 9 & -7.7 & $-14.3 * *$ \\
\hline 07X14 & 107 & -0.5 & -0.9 & 44 & $9.3^{*}$ & $27.5^{* *}$ & 10 & 2.6 & 0.0 \\
\hline 08X15 & 108 & -2.3 & -2.7 & 32.5 & 4.0 & 8.3 & 11 & $7.3^{*}$ & 4.8 \\
\hline 09X16 & 116 & 1.8 & -1.7 & 28 & $11.8^{* *}$ & $21.1^{* *}$ & 10.5 & -2.3 & -4.5 \\
\hline $10 \times 17$ & 111 & 0.9 & -1.8 & 26.5 & $30.9 * *$ & $-14.1^{* *}$ & 11 & -4.3 & $-15.4^{* *}$ \\
\hline I1X12 & 103 & $7.2 * *$ & $-7.2 * *$ & 38 & -3.2 & -8.4 & 9.5 & $-7.3 *$ & $-13.6 * *$ \\
\hline $18 \times 25$ & 107 & 0.5 & $-6.1 * *$ & 39.5 & $12.9 * *$ & 3.9 & 9.5 & 0.0 & -5.0 \\
\hline 19X26 & 107 & -0.5 & $-2.7 *$ & 46 & 5.1 & -3.2 & 9.5 & $18.8^{* *}$ & $18.8^{* *}$ \\
\hline $20 \times 27$ & 103 & $-5.1^{* *}$ & $-7.2 * *$ & 32 & $-11.7 * *$ & $-12.3^{*}$ & 11.5 & $7.0^{*}$ & 4.5 \\
\hline $21 X 28$ & 106 & $-3.6^{* *}$ & $-3.6^{* *}$ & 42 & $7.7^{*}$ & $23.5^{* *}$ & 10.5 & $10.5^{* *}$ & 5.0 \\
\hline $22 \times 29$ & 94 & $-13.4^{* *}$ & $-13.8 * *$ & 38 & 4.1 & 5.6 & 10.5 & $23.5^{* *}$ & $23.5^{* *}$ \\
\hline $23 \times 30$ & 110 & $-4.3^{* *}$ & $-6.8^{* *}$ & 30 & $18.8^{* *}$ & $-10.4^{*}$ & 11 & 5.3 & $-31.3^{* *}$ \\
\hline $24 X 31$ & 103 & $-5.1^{* *}$ & $-5.5 * *$ & 40 & $8.8^{*}$ & $14.3^{* *}$ & 10 & 5.3 & -4.8 \\
\hline BGN06 & 109 & & & 36.5 & & & 10.5 & & \\
\hline BGN07 & 107 & & & 46 & & & 9.5 & & \\
\hline BGN08 & 111 & & & 32.5 & & & 10 & & \\
\hline BGN09 & 110 & & & 28 & & & 11 & & \\
\hline BGN10 & 113 & & & 25 & & & 10 & & \\
\hline BGN11 & 111 & & & 37 & & & 11 & & \\
\hline BGN12 & 111 & & & 41.5 & & & 9.5 & & \\
\hline
\end{tabular}


Table 1. Cont'd.

\begin{tabular}{|c|c|c|c|c|c|c|c|c|c|}
\hline \multirow{2}{*}{$\begin{array}{l}\text { Crosses/ } \\
\text { Parents }\end{array}$} & \multirow{2}{*}{$\begin{array}{l}\text { Mean } \\
\text { performancee } \\
\text { (days) }\end{array}$} & \multicolumn{2}{|c|}{ Days to first harvest } & \multirow{2}{*}{$\begin{array}{l}\text { Mean } \\
\text { performance } \\
(\mathrm{cm})\end{array}$} & \multicolumn{2}{|c|}{ Fruit length } & \multirow{2}{*}{$\begin{array}{c}\text { Mean } \\
\text { performance } \\
(\mathrm{cm})\end{array}$} & \multicolumn{2}{|c|}{ Fruit diameter } \\
\hline & & $\begin{array}{c}\text { Mid } \\
\text { parent }\end{array}$ & $\begin{array}{l}\text { Better } \\
\text { parent }\end{array}$ & & $\begin{array}{c}\text { Mid } \\
\text { parent }\end{array}$ & $\begin{array}{l}\text { Better } \\
\text { parent }\end{array}$ & & $\begin{array}{c}\text { Mid } \\
\text { parent }\end{array}$ & $\begin{array}{l}\text { Better } \\
\text { parent }\end{array}$ \\
\hline BGN13 & 114 & & & 39 & & & 9 & & \\
\hline BGN14 & 108 & & & 34.5 & & & 10 & & \\
\hline BGN15 & 110 & & & 30 & & & 10.5 & & \\
\hline BGN16 & 118 & & & 35.5 & & & 10.5 & & \\
\hline BGN17 & 107 & & & 15.5 & & & 13 & & \\
\hline BGN18 & 114 & & & 32 & & & 10 & & \\
\hline BGNI9 & 110 & & & 35 & & & 10.5 & & \\
\hline BGN20 & 111 & & & 36 & & & 11 & & \\
\hline BGN2I & 110 & & & 44 & & & 9 & & \\
\hline BGN22 & 109 & & & 37 & & & 8.5 & & \\
\hline BGN23 & 118 & & & 17 & & & 16 & & \\
\hline BGN24 & 109 & & & 38.5 & & & 8.5 & & \\
\hline BGN25 & 99 & & & 38 & & & 9 & & \\
\hline BGN26 & 105 & & & 47.5 & & & 8 & & \\
\hline BGN27 & 106 & & & 36.5 & & & 10.5 & & \\
\hline BGN28 & 110 & & & 34 & & & 10 & & \\
\hline BGN29 & 108 & & & 36 & & & 8.5 & & \\
\hline BGN3O & 112 & & & 33.5 & & & 11 & & \\
\hline BGN3I & 108 & & & 35 & & & 10.5 & & \\
\hline $\begin{array}{l}\mathrm{SE} \\
\end{array}$ & & 1.1 & 1.0 & & 3.2 & 4.1 & & 3.1 & 4.1 \\
\hline LSD (0.05) & & 2.4 & 2.2 & & 7.0 & 8.9 & & 6.8 & 8.9 \\
\hline LSD (0.01) & & 3.4 & 3.1 & & 9.8 & 12.5 & & 9.5 & 12.5 \\
\hline
\end{tabular}


Table 1. Cont'd.

\begin{tabular}{|c|c|c|c|c|c|c|c|c|c|}
\hline \multirow[t]{2}{*}{$\begin{array}{l}\text { Crosses/ } \\
\text { Parents }\end{array}$} & \multirow{2}{*}{$\begin{array}{c}\text { Mean } \\
\text { performance } \\
\text { (number) }\end{array}$} & \multicolumn{2}{|c|}{ No. of fruits/plant } & \multirow{2}{*}{$\begin{array}{c}\text { Mean } \\
\text { performance } \\
(\mathrm{kg})\end{array}$} & \multicolumn{2}{|c|}{$\begin{array}{l}\text { Individual fruit } \\
\text { weight }\end{array}$} & \multirow{2}{*}{$\begin{array}{c}\text { Mean } \\
\text { performance } \\
(\mathrm{kg})\end{array}$} & \multicolumn{2}{|c|}{ Yield/plant } \\
\hline & & $\begin{array}{c}\text { Mid } \\
\text { parent }\end{array}$ & $\begin{array}{l}\text { Better } \\
\text { parent }\end{array}$ & & $\begin{array}{c}\text { Mid } \\
\text { parent }\end{array}$ & $\begin{array}{l}\text { Better } \\
\text { parent }\end{array}$ & & $\begin{array}{c}\text { Mid } \\
\text { parent }\end{array}$ & $\begin{array}{l}\text { Better } \\
\text { parent }\end{array}$ \\
\hline $06 \times 13$ & 10 & 25.I1 & 11.1 & 1.7 & 0.0 & 0.0 & 17.0 & 25.0 & 11.1 \\
\hline 07X14 & 11 & $29.4^{* *}$ & $22.2^{*}$ & 2.1 & $20.1^{* *}$ & 5.0 & 23.1 & $69.5^{* *}$ & $44.4^{* *}$ \\
\hline 08X15 & 7 & 16.7 & $16.7 * *$ & 1.8 & 5.9 & 5.9 & 12.6 & 23.5 & $23.5^{*}$ \\
\hline 09X16 & 6 & 9.1 & 0.0 & 1.5 & -14.3 & $-16.7^{* *}$ & 9.0 & -6.7 & -16.7 \\
\hline $10 \times 17$ & 10 & $53.8^{*}$ & 42.9 & 1.8 & $12.5^{* *}$ & $12.5^{* *}$ & 18.0 & $73.1^{* *}$ & $60.7^{* *}$ \\
\hline IlXI2 & 7 & -6.7 & -22.2 & 1.8 & -2.7 & -10.0 & 12.6 & -7.7 & -17.6 \\
\hline $18 X 25$ & 9 & 12.5 & 12.4 & 1.7 & 9.7 & -5.6 & 15.3 & $23.4^{* *}$ & 6.2 \\
\hline $19 \times 26$ & 11 & 15.8 & 0.0 & 2.0 & $12,0^{* *}$ & 11.1 & 22.0 & $67.9^{* *}$ & $61.8^{* *}$ \\
\hline $20 \times 27$ & 6 & -18.9 & $-23.1^{* *}$ & 2.0 & 5.3 & 0.0 & 12.0 & -14.9 & $-23.1^{*}$ \\
\hline $21 X 28$ & 11 & $22.2^{* *}$ & $22.2 * *$ & 2.0 & $17.6^{* *}$ & $17.6^{* *}$ & 22.0 & $52.2^{* *}$ & $43.8^{* *}$ \\
\hline $22 \times 29$ & 9 & $28.6^{* *}$ & $28.6^{* *}$ & 1.6 & 3.2 & 0.0 & 14.4 & $32.7^{* *}$ & $28.6^{* *}$ \\
\hline $23 \times 30$ & 9 & $20.0 * *$ & $12.6^{*}$ & 1.8 & 5.9 & 0.0 & 16.2 & $26.6^{* *}$ & 12.5 \\
\hline $24 \times 31$ & 9 & 9.1 & 0.0 & 2.1 & $40.0^{* *}$ & $40.0^{* *}$ & 18.9 & $52.7^{* *}$ & $40.1^{* *}$ \\
\hline BGN06 & 7 & & & 1.7 & & & 11.9 & & \\
\hline BGN07 & 8 & & & 2.0 & & & 16.0 & & \\
\hline BGN08 & 6 & & & 1.7 & & & 10.2 & & \\
\hline BGN09 & 5 & & & 1.7 & & & 8.5 & & \\
\hline BGN10 & 6 & & & 1.6 & & & 9.6 & & \\
\hline BGN11 & 6 & & & 2.0 & & & 12.0 & & \\
\hline BGN12 & 9 & & & 1.7 & & & 15.3 & & \\
\hline
\end{tabular}


Table 1. Cont'd.

\begin{tabular}{|c|c|c|c|c|c|c|c|c|c|}
\hline \multirow[t]{2}{*}{$\begin{array}{l}\text { Crosses/ } \\
\text { Parents }\end{array}$} & \multirow{2}{*}{$\begin{array}{c}\text { Mean } \\
\text { performance } \\
\text { (number) }\end{array}$} & \multicolumn{2}{|c|}{ No. of fruits/plant } & \multirow{2}{*}{$\begin{array}{c}\text { Mean } \\
\text { performance } \\
(\mathrm{kg})\end{array}$} & \multicolumn{2}{|c|}{$\begin{array}{l}\text { Individual fruit } \\
\text { weight }\end{array}$} & \multirow{2}{*}{$\begin{array}{c}\text { Mean } \\
\text { performance } \\
(\mathrm{kg})\end{array}$} & \multicolumn{2}{|c|}{ Yield/plant } \\
\hline & & $\begin{array}{c}\text { Mid } \\
\text { parent }\end{array}$ & $\begin{array}{l}\text { Better } \\
\text { parent }\end{array}$ & & $\begin{array}{c}\text { Mid } \\
\text { parent }\end{array}$ & $\begin{array}{l}\text { Better } \\
\text { parent }\end{array}$ & & $\begin{array}{c}\text { Mid } \\
\text { parent }\end{array}$ & $\begin{array}{l}\text { Better } \\
\text { parent }\end{array}$ \\
\hline BGN13 & 9 & & & 1.7 & & & 15.3 & & \\
\hline BGN14 & 7.5 & & & 1.5 & & & 11.3 & & \\
\hline BGN15 & 6 & & & 1.7 & & & 10.2 & & \\
\hline BGN16 & 6 & & & 1.8 & & & 10.8 & & \\
\hline BGN17 & 7 & & & 1.6 & & & 11.2 & & \\
\hline B0N18 & 8 & & & 1.8 & & & 14.4 & & \\
\hline BGN19 & 7 & & & 1.8 & & & 12.6 & & \\
\hline BGN20 & 7.8 & & & 2.0 & & & 15.6 & & \\
\hline BGN21 & 9 & & & 1.7 & & & 15.3 & & \\
\hline BGN22 & 7 & & & 1.6 & & & 11.2 & & \\
\hline B0N23 & 7 & & & 1.6 & & & 11.2 & & \\
\hline BGN24 & 9 & & & 1.5 & & & 13.5 & & \\
\hline BGN25 & 8 & & & 1.3 & & & 10.4 & & \\
\hline BGN26 & 8 & & & 1.7 & & & 13.6 & & \\
\hline BGN27 & 7 & & & 1.8 & & & 12.6 & & \\
\hline BGN28 & 8 & & & 1.7 & & & 13.6 & & \\
\hline B0N29 & 7 & & & 1.5 & & & 10.5 & & \\
\hline BGN30 & 8 & & & 1.8 & & & 14.4 & & \\
\hline BGN31 & 7.5 & & & 1.5 & & & 11.3 & & \\
\hline SE & & 4.9 & 5.2 & & 3.6 & 3.9 & & 8.3 & 8.0 \\
\hline LSD (0.05) & & 10.7 & 11.3 & & 7.8 & 8.5 & & 18.1 & 17.4 \\
\hline $\operatorname{LSD}(0.0 \mathrm{l})$ & & 15.0 & 15.9 & & 11.0 & 11.9 & & 25.4 & 24.4 \\
\hline
\end{tabular}


Eight crosses showed significant positive mid parent heterosis for number of fruits per plant and range was 12.5-53.8 percent. The highest significant positive heterosis was obtained by the cross 10 x 17 followed by 7 x 14 (29.4\%). Seven crosses exhibited significant positive better parent heterosis for this trait. The significant positive better parent heterosis range from 12.4-42.9\%. The highest significant positive better parent heterosis was also attained by the cross $10 \times 17$ followed by 22 x 29 (28.6\%) and Singh et al. (1998) observed higher percentage of heterosis of number of fruits in bottle gourd. Similar results were also reported by Sit and Sirohi (2002).

In case of individual fruit weight, two crosses showed more than $20 \%$ mid parent heterosis and highest significant positive heterosis was obtained by the cross $24 \times 31(40.0 \%)$, whereas only one cross $24 \times 31$ (40.0\%) exhibited more than $20 \%$ better parent heterosis. Significant positive heterosis for fruit weight in bottle gourd was also reported by Singh et al. (1998) and Sit and Sirohi (2002).

Ten crosses for yield per plant showed significant positive mid parent heterosis. Percent of mid parent positive heterosis range from 23.4 to $73.1 \%$. The highest significant positive heterosis was obtained from the cross $10 \mathrm{x} 17$. More than $50 \%$ heterosis was observed from four crosses. The highest were 10 x 17 (73.1\%), 7 x 14 (69.5\%), 24.31 (52.7 \%), and 21 x 28 (52.2\%). Kumar et al. (1999) reported $117.26 \%$ yield increase over mid parent in bottle gourd. In the case of better parent heterosis for this trait, seven crosses showed significant positive better parent heterosis. Percent of significant positive heterosis ranged from 23.5 to $61.8 \%$. The highest significant positive better parent heterosis was exhibited from the hybrid $19 \times 26$ followed by $10 \times 17$ (60.7\%). Janakiram and Sirohi (1992) reported the best performing hybrids for yield.

Janakiram and Sirohi (1989) concluded that the increase in yields of hybrids was mainly due to fruits number per plant, single fruit weight and fruit size, while Singh et el. (1998) reported that heterosis in yield was attributed to earliness of fruit harvest. In case of yield per plant, the cross combination $10 \times 17$ and $19 \times 26$ manifested the highest heterosis over mid parent and better parent, respectively, but not in all characters. So it could be concluded that heterosis was not responsible to only fruit length or fruit diameter, but also responsible to individual fruit weight, number of fruits per plant and earliness.

\section{References}

Anonymous. 2004. Year Book of Agricultural Statistics of Bangladesh 2002. Bangladesh Bureau of Statistics, Ministry of Planning, Government of People's Republic of Bangladesh, Dhaka, Bangladesh. pp 94-102

Janakiram. T and P.S. Sirohi. 1992. Studies on heterosis on quantitive characters in bottle gourd. J. Maharashtra Agril. Univ. 17(2): 204-206. 
Janakiram, T and P.S. Sirohi. 1989. Hetrosis studies in round-fruited bottle gourd. J. Maharashtra Agril. 76 (6): 339-342.

Kumar Sarvesh, S.P., R. C. Singh, Jaiswal and S. Kumar. 1999. Heterosis over mid and top parent under the line x tester fashion in bottle gourd. Veg. Sc. 26(1): 30-32.

Singh. P.K., J.C. Kumar and J.R. Sharma. 1998. Heterosis studies in long fruited bottle gourd. Vegetable Science 25(1): 55-57.

Sit. A.K and P.S. Sirohi. 2002. Exploitation of heterosis in bottle gourd. Hort. J. 15(2): 55-60. 